\title{
Early introduction of solid foods and the low prevalence of food allergy and atopic eczema among preterm children during the first year of life
}

\author{
Jaakko Yrjänä ${ }^{1,2^{*}}$, Marita Valkama ${ }^{1}$, Petri Kulmala ${ }^{1,2}$ \\ From Food Allergy and Anaphylaxis Meeting 2014 \\ Dublin, Ireland. 9-11 October 2014
}

\section{Background}

Clinical experience indicates preterm children having less food allergies (FA) and atopic dermatitis (AD) than fullterm children. This is despite the fact that solid foods are usually started earlier in preterm infants to ensure the growth and development.

\section{Methods}

We aimed to study early nutrition, FA and $\mathrm{AD}$ in a retrospective study. All the preterm infants $(<37$ gestational weeks) born and having been regularly followed up at the Department of Pediatrics, Oulu University Hospital during the years 2008-2012 are included. 383 preterm infants were identified from the hospital patient registry. Followup data from outpatient clinic visits until the age of 1 year was available in $31 / 157$ (20\%) of preterm (33-37 gestational weeks), 136/177 (77\%) of very preterm (28-32 gestational weeks) and $42 / 49$ (86\%) of extremely preterm ( $<28$ gestational weeks) infants, and these 209 children were included in the analyses. The frequencies of FA and AD were compared to our survey on infants from the general population.

\section{Results}

The prevalence of FA and AD among 248 infants from the general population were $4.8 \%$ and $12.9 \%$, respectively. In comparison, 4 (1.9\%) of the all 209 preterm children had a confirmed diagnosis of FA $(\mathrm{p}=0.12)$ and $5(2.4 \%)$ AD $(\mathrm{p}=0.0001)$. Among the very preterm infants only $1(0.7 \%)$ had FA ( $\mathrm{p}=0.038)$ and $4(2.9 \%)$ had $\mathrm{AD}(\mathrm{p}=0.001)$. No FA $(\mathrm{p}=0.23)$ or $\mathrm{AD}(\mathrm{p}=0.007)$ were diagnosed among the extremely preterm infants by the

age of 1 year. As calculated from the expected time of labor the mean age at the first introduction of fruits, vegetables, meat and grain to the diet in all preterm infants were $0.9,1.0,2.2$ and 3.6 months, respectively. These mean ages among the very preterm babies were $0.5,0.6,1.9$ and 3.2 months, and among the extremely preterm babies $0.02,0.1,1.3$ and 2.8 months, respectively.

\section{Conclusion}

Solid foods were introduced to the diet of preterm infants very early. However, the prevalence of FA and $\mathrm{AD}$ were low. These findings will be verified in a larger study population. Furthermore, a prospective study is planned to investigate relationship between early nutrition and prevalence of atopic disease in preterm infants.

\section{Authors' details}

${ }^{1}$ Department of Pediatrics, Medical Research Center Oulu, Oulu University Hospital and University of Oulu, Oulu, Finland. 'Institute of Diagnostics, Department of Medical Microbiology and Immunology, Medical Research Center Oulu and University of Oulu, Oulu, Finland.

Published: 30 March 2015

\section{doi:10.1186/2045-7022-5-S3-O1}

Cite this article as: Yrjänä et al:: Early introduction of solid foods and the low prevalence of food allergy and atopic eczema among preterm children during the first year of life. Clinical and Translational Allergy 2015 5(Suppl 3):O1. 